I Universidade Federal do Rio de Janeiro (UFRJ), Programa de Pós-Graduação

em Antropologia Social, Museu Nacional, Rio de Janeiro, RJ, Brasil

vivianemf@gmail.com

https://orcid.org/0000-0002-0399-7139

Viviane Fernandes'

\title{
DA EDUCAÇÃO AO EMPURRÃO: A PARTICIPAÇÃO DAS CIÊNCIAS COMPORTAMENTAIS EM PROGRAMAS DE EDUCAÇÃO FINANCEIRA
}

Em dezembro de 20I4, participei, pela primeira vez, da Conferência de Educação Financeira e Comportamento do Investidor. O evento, que se destacava como um dos principais encontros sobre o tema da educação financeira no país, era promovido pela Comissão de Valores Imobiliários (CVM) e contava com o suporte de mais duas instituições do sistema financeiro nacional: a Associação Brasileira das Entidades dos Mercados Financeiro e de Capitais (Anbima) e a BM\&FBovespa, ${ }^{\mathrm{I}}$ além de receber apoio da Organização para a Cooperação e Desenvolvimento Econômico (OCDE).

Em um grande salão de convenção de um reconhecido hotel da Zona Sul do Rio de Janeiro, reuniam-se mais de 500 pessoas - público bastante equilibrado entre homens e mulheres e segmentado em diferentes faixas etárias. A plateia era composta pelos profissionais das instituições organizadoras, consultores e analistas financeiros, professores, estudantes, jornalistas, investidores e servidores públicos. A considerar os crachás de identificação dos participantes, podiam-se distinguir dois grupos, um composto por especialistas em investimentos e mercado de capitais e outro, mais heterogêneo, formado por aqueles interessados nas inciativas de educação financeira - no qual eu me incluía.

O que reunia essas pessoas na ocasião eram as discussões sobre a maneira como as decisões financeiras eram tomadas. Como as pessoas decidem onde investir? Como avaliam o que consumir e o que poupar? 
Como administram os recursos financeiros? Como desenvolvem a capacidade de planejar? Especialistas e acadêmicos de diferentes áreas foram chamados para expor recentes experiências e pesquisas que trouxessem respostas a essas questões. Gestores de políticas públicas, consultores independentes ou profissionais de empresas privadas ligados ao universo financeiro buscavam conhecer o comportamento humano no que se refere ao uso do dinheiro para desenvolver formas de "melhorar" a maneira como as pessoas organizam suas finanças, formando possíveis investidores. ${ }^{2}$

Interessada em estudar a Estratégia Nacional de Educação Financeira (Enef), ${ }^{3}$ havia me inscrito na Conferência com intuito de compreender como ocorria, na prática, o desenvolvimento dessa política. Minha principal intenção, nesse início de trabalho de campo, era conhecer os programas de educação financeira, seus atores e público-alvo. Em especial, buscava entender como essas iniciativas se desdobravam para além das páginas do plano diretor de uma política pública. ${ }^{4}$

Tendo em vista que a Enef visava ampliar a compreensão das pessoas acerca dos conceitos e produtos financeiros, desenvolvendo competências para que elas aprimorassem suas escolhas financeiras e soubessem administrar seus recursos, imaginava que os economistas e as teorias econômicas ocupariam o centro das discussões. Ao longo da Conferência, entretanto, observei que muitos dos painéis eram conduzidos por psicólogos e neurocientistas, e, nesse sentido, eram as discussões sobre o comportamento das pessoas e o funcionamento do cérebro que ganhavam relevância.

A plateia mostrava-se atenta ao acompanhar as imagens coloridas que destacam ora o hemisfério esquerdo do cérebro, ora o direito. Modelos de tomada de decisão eram apresentados junto com as regiões do cérebro acionadas na execução de determinada atividade. Como explicado por um palestrante, as pesquisas em neurofinanças haviam se desenvolvidos recentemente. Com a ajuda de técnicas menos invasivas e novas ferramentas, passou a ser possível "desvendar o cérebro" e não mais considerá-lo uma "caixa-preta". A ressonância magnética funcional ou o eletroencefalograma associado aos jogos de simulação e às análises estatísticas traziam novas formas de acessar o que antes parecia inacessível. Era a partir das atividades cerebrais que a tomada de decisão e comportamentos financeiros poderiam ser explicados. ${ }^{5}$ 


\section{Are winners using emotion?}

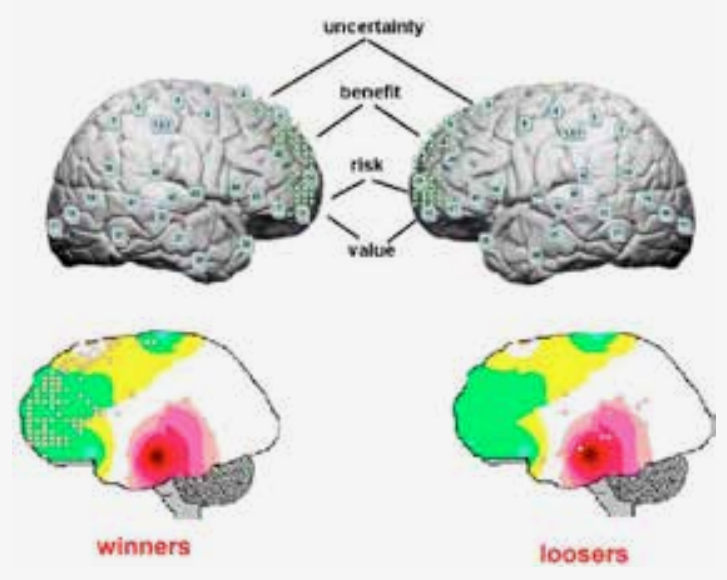

Figura I

Exemplo de imagens transmitidas nas apresentações

Fonte: iecbrazil.com.br

Tomo a Conferência de 20I4 como ponto de observação privilegiado para descrever o modo como as ciências comportamentais passaram a integrar-se à política de educação financeira. Os registros desse evento permitem destacar o momento em que saberes relativos ao funcionamento do cérebro e propensões comportamentais são apresentados e legitimados como relevantes contribuições para aprimorar as iniciativas de educação financeira. Diferentemente do que imaginava, as principais discussões da Conferência de Educação Financeira e Comportamento do Investidor não se voltavam aos conteúdos e técnicas que envolveriam um projeto pedagógico em finanças (aprofundando-se em temas como finanças pessoais; técnicas de orçamento e de planejamento; avaliações de investimentos em previdência social e privada; ou mesmo instruções sobre o sistema financeiro nacional) - estavam em discussão, principalmente, as emoções, os comportamentos e a racionalidade humana. Grande parte do enfoque apoiava-se no funcionamento do cérebro e, consequentemente, no argumento de que conhecer o funcionamento desse órgão poderia auxiliar a construção de intervenções mais eficazes para o ajuste das condutas das pessoas, em especial na melhoria da tomada de decisões financeiras.

Esse texto, apresenta-se assim, como um registro de pesquisa que se propõe explorar em que contexto as ciências comportamentais ganham espaço dentro das iniciativas de educação financeira desenvolvidas no país. A 
partir de uma abordagem etnográfica, busco demonstrar a importância da Conferência de Educação Financeira e Comportamento do Investidor como ferramenta para reunir agentes dispersos e conferir legitimidade e congruência a um conjunto de teorias que - na intersecção da psicologia e economia - discutem os mecanismos da tomada de decisões econômicas.

Dividido em três partes, o artigo começa como uma rápida descrição da Enef para, em seguida, apresentar como essa política, incialmente pautada em pedagogias relacionadas à administração dos orçamentos pessoais e familiares, adquire novos contornos mediante mudanças na concepção $\mathrm{da}(\mathrm{s})$ racionalidade(s) dos agentes. $\mathrm{Na}$ última seção, recupero as críticas de Polanyi sobre o conceito de Homo oeconomicus com o intuito de refletir sobre os modelos de intervenção propostos pelas ciências comportamentais.

\section{UMA POLÍTICA DE EDUCAÇÃO FINANCEIRA}

A educação financeira é o processo mediante o qual os indivíduos e as sociedades melhoram sua compreensão dos conceitos e dos produtos financeiros, de maneira que, com informação, formação e orientação claras, adquiram os valores e as competências necessários para se tornarem conscientes das oportunidades e dos riscos neles envolvidos e, então, façam escolhas bem informados, saibam onde procurar ajuda, adotem outras ações que melhorem o seu bem-estar, contribuindo, assim, de modo consistente para formação de indivíduos e sociedades responsáveis, comprometidos com o futuro (Brasil, 2010: 20).

Inspirado no conceito de educação financeira promovido pela OCDE, o governo brasileiro, desde 2010 , vem construindo iniciativas próprias a favor do desenvolvimento de competências financeiras nas pessoas. Considerada pelos representantes do Sistema Financeiro Nacional um tema urgente, a Enef foi decretada política pública de Estado com o objetivo de auxiliar as pessoas a estar mais preparadas para tomar decisões financeiras.

Entre os elaboradores da Enef ${ }^{6}$ havia o entendimento de que, nos últimos tempos, muitos fatores confluíam de forma a tornar mais difícil a decisão das pessoas sobre quais serviços e ferramentas utilizar, entre eles: o aumento do número de instituições que passaram a oferecer produtos e serviços financeiros; a grande variedade e também semelhanças entre os produtos; e a maior complexidade dos instrumentos, fazendo-se necessárias habilidades específicas para a compreensão das informações prestadas pelas instituições, bem como para análise dos custos e riscos das contratações. Diante desse cenário, é oferecido, em resposta, um modelo pedagógico elaborado para a redução dos riscos que decisões de consumo ou de aplicações financeiras não adequadas podem trazer para o futuro do próprio indivíduo ou de sua família (Soares, 2017; Fernandes, 2019).

Outros dois motivos, todavia, parecem essenciais para a compreensão das preocupações do governo brasileiro com a educação financeira: o pri- 
meiro deles aborda o envelhecimento da população, considerando um significativo incremento dos gastos com a saúde; o segundo considera a redução da taxa de natalidade, fator que, no futuro, implicaria menor número de trabalhadores e maior camada da população aposentada, elementos que, nesse caso, são vistos como desafios para a previdência social.

Há, assim, grande interesse por parte dos governos de que as pessoas tenham poupança, saibam fazer investimentos e acompanhem a gestão de seus recursos ao longo da vida. Diante desse desafio, diferentes inciativas de educação financeira foram colocadas em prática, tanto para crianças e adolescentes ${ }^{7}$ quanto para o público adulto. A Conferência era mais uma dessas iniciativas - a partir de sua primeira edição, em 2013 , tornou-se evento anual, relevante não apenas por mobilizar um grande número de pessoas e legitimar um conjunto de saberes, mas, principalmente, por promover o compartilhamento de experiências entre a academia e agentes responsáveis pelo desenvolvimento de programas tanto no setor público quanto no privado. ${ }^{8}$

\section{AS CIÊNCIAS COMPORTAMENTAIS E AS CRÍTICAS AO HOMO OECONOMICUS}

Para os que trabalhavam com o tema da educação financeira, a Conferência havia se tornado um evento aguardado. Sua inscrição gratuita e aberta a todos os interessados favorecia o encontro de uma grande quantidade de pessoas de diferentes estados do país, além de integrar como palestrantes um conjunto de especialistas (profissionais de mercado ou acadêmicos) com atuação internacional. 9

Para os educadores e consultores financeiros - os quais pude acompanhar durante o trabalho de campo - a Conferência tinha um papel de formação: era o momento de conhecer os recentes estudos de educação financeira, identificar tendências, informar-se sobre novos conceitos e as principais discussões ligadas à temática. No entanto, não apenas o conteúdo das palestras era atrativo ao público participante; a oportunidade de interação produzida pelo encontro também era valorizada como ocasião para circular, conhecer pessoas e se relacionar com outros profissionais da área, ${ }^{\text {Io }}$ oportunidade ainda para divulgar seu próprio trabalho e conhecer os variados projetos em andamento. ${ }^{\text {I }}$

$\mathrm{Na}$ edição de 20I4, o discurso de abertura, proferido pelo presidente da CVM, anunciava o caráter interdisciplinar do encontro e convidava a plateia a manter "as cabeças abertas". Nas boas-vindas ao evento, era pedido aos participantes que se mantivessem receptivos às novas teorias que seriam apresentadas. Em seguida, era sugerido que a incorporação dos saberes das ciências comportamentais aos programas de educação financeiras seria uma oportunidade de "pensar fora do quadrado".

Os painéis e as discussões subsequentes apresentaram estudos oriundos da economia comportamental, neuroeconomia e psicologia econômica. 
As diferentes abordagens teóricas e metodológicas dessas áreas, no entanto, eram reunidas e apresentadas ao longo da Conferência sob o guarda-chuva das ciências comportamentais. Esse campo de pesquisa era, assim, alargado e tornava-se capaz de acolher diferentes estudos, sejam eles relacionados à psicologia, psicologia econômica, neurociência, às neurofinanças, economia comportamental, economia cognitiva ou até mesmo à sociologia, antropologia e ao marketing.

Pesquisas sobre o funcionamento do cérebro ganhavam destaque nesse encontro principalmente pelo objetivo perseguido pela CVM de ampliar o número de poupadores no país. Uma vez que entre as metas da instituição estava o desenvolvimento da formação de poupança, uma pergunta surgia como incontornável aos reguladores e analistas financeiros: “por que as pessoas não poupam?". Logo, na tentativa de responder a esse questionamento fazia-se a aposta em estudos sobre a escolha humana, visando, em seguida, traduzir os achados em intervenções práticas - ações que, ao final, garantissem mudanças nos comportamentos econômicos das pessoas.

Era nesse sentido que os aportes trazidos pelas ciências comportamentais surgiam como um novo recurso para as iniciativas de educação financeira. Frente aos questionamentos dos resultados obtidos com a avaliação dos programas executados, conformava-se a ideia de que apenas a oferta de informações não se mostrava suficiente para que as pessoas absorvessem o conteúdo transmitido e transformassem conhecimento em novos comportamentos. Pesquisas ponderavam o alcance dos programas e apontavam que a própria passagem do tempo levaria as pessoas a perder a motivação de manter as práticas financeiras aprendidas (Fernandes, Lynch \& Netemeyer, 20I4; Kaiser \& Menkhoff, 20I6). Enquanto alguns autores argumentavam que as intervenções pareciam se correlacionar muito pouco com a melhoria dos conhecimentos e habilidades financeiras, outros defendiam que políticas educativas alcançavam apenas alguns de seus objetivos e seria exigido mais tempo para produzir os efeitos esperados (Lusardi, 2003; Miller et al., 20I5).

A validade de um modelo pedagógico pautado em um agente racional que reúne informações, planeja e calcula suas opções para a tomada de decisões passou a ser contestada. Uma vez que controvérsias colocavam em dúvida a capacidade preditiva de teorias econômicas relacionadas à tomada de decisão, abria-se espaço para pesquisas que procuravam compreender empiricamente os processos de escolha. Diante das críticas, o método empírico das ciências comportamentais ganhava mais atenção e mostrava-se como um caminho para se conhecer melhor o agente-alvo da política pública e, consequentemente, melhorar as abordagens dos programas. Pesquisas em psicologia e economia comportamental identificavam uma série de li- 
mitações cognitivas que atuava de forma a impedir que os agentes tomassem decisões racionais. Esses estudos foram apresentados como capazes de melhorar os modelos econômicos por incorporar a psicologia.

$\mathrm{Na}$ intersecção entre economia e psicologia, as pesquisas de Herbert Simon (I955, I982) sobre a "racionalidade limitada" são consideradas percursoras da economia comportamental. O autor, interessado na escolha humana, argumenta que não haveria como predizer exatamente como as pessoas se comportam e insiste que são desconhecidas as variáveis relevantes para a compreensão das decisões, por exemplo, quais informações são privilegiadas ou quais são os interesses particulares que motivam os agentes. Amos Tversky e Daniel Kahneman (1974, I979), mais tarde, demonstraram que, em situações complexas, mesmo os agentes detentores de informações poderiam cometer erros de escolha. A partir de uma série de experimentos, os autores buscaram evidenciar que a intuição poderia fazer com que as pessoas tomassem decisões consideradas erradas. Richard Thaler, por sua vez, amplia as críticas ao Homo oeconomicus, argumentando sobre a importância do contexto no processo de escolha (Thaler, I980, I999; Thaler \& Shefrin, I98I). Ao destacar o contexto, o autor aponta para a possibilidade de influenciar decisões a partir do modo como as escolhas são apresentadas. Como argumenta, mesmo os detalhes aparentemente insignificantes podem ter impacto no comportamento das pessoas - esses detalhes poderiam ser usados para desviar o foco ou atrair a atenção para determinadas direções (Thaler \& Sunstein, 2008).

Logo, diferentemente do que se aceitava, os indivíduos não deveriam ser considerados perfeitamente racionais ou capazes de utilizar toda a informação disponível em seus processos decisórios. O modelo teórico fundamentado em um indivíduo racional, egoísta, que toma decisões com base em cálculos de custo/benefício (Jevons, I875; Friedman, I957), tornava-se alvo de críticas, mesmo nos casos em que fosse considerado apenas uma aproximação fictícia, um modelo de análise.

Ao apresentar uma linguagem simples e acessível para falar da mente, a ciência ou economia comportamental concentra-se especialmente na noção de "vieses cognitivos". Em outras palavras, as pessoas teriam "atalhos mentais", isto é, formas abreviadas de tomar decisões - característica essa que as faz economizar tempo na realização de suas escolhas, mas que também pode levar a erros de avaliação (Tversky \& Kahneman, I974). Uma vez que as pessoas precisam tomar muitas decisões por dia, o cérebro disporia de um sistema para fazer escolhas rápidas, às vezes automáticas e com economia de esforço (Kahneman, 20I I). No entanto, apesar de as heurísticas (nome dado a esses atalhos utilizados para perceber e avaliar dados) serem mecanismos efetivos e importantes no funcionamento do cérebro, em cenários mais complexos, elas também poderiam ser fonte de erros. Chama- 
dos de vieses, esses erros sistemáticos, em circunstâncias particulares, se repetiriam de forma previsível.

Para corrigir os vieses de comportamento, uma das principais ferramentas das ciências comportamentais é a utilização de nudges, isto é, a aplicação de pequenos estímulos para tentar ajustar condutas. Uma vez que as decisões humanas são falhas e tendenciosas, esses estímulos seriam usados de forma sutil para encorajar determinados comportamentos ou remover barreiras capazes de impedir certas decisões. Logo, o nudge não seria uma ordem, mas um ajuste na "arquitetura de escolha" com a intenção de exercer influência (Thaler \& Sunstein, 2008). Conhecido como "empurrãozinho" ou "cutucão", os nudges teriam a capacidade de orientar as pessoas para determinadas direções, procurando influenciar o comportamento. Avisos sobre o consumo de calorias, lembretes sobre uma consulta médica, mensagem sobre o vencimento de uma fatura, a inscrição automática em um plano de previdência, a configuração-padrão de um celular, o design de um site que apresenta determinadas informações em fontes maiores são exemplos de nudges (Sunstein, 20I4). Entre os diferenciais dessa solução - que vem atraindo a atenção de instituições públicas e privadas

- estaria o baixo custo de implementação frente ao potencial para obter resultados em diferentes áreas, aplicável aos mais variados objetivos, sejam eles ligados à saúde, educação, alimentação ou ecologia.

A crítica ao Homo oeconomicus e a ênfase na complexidade da biologia humana marcam a mudança na forma de conceber o processo de escolha dos indivíduos e, consequentemente, na forma como as ciências comportamentais propõem intervir para "aprimorar" as decisões. Isso posto, torna-se relevante investigar qual o modelo de agente econômico que passa a vigorar como alvo das políticas baseadas nesses novos conceitos, ferramentas e abordagens.

\section{CORRIGINDO VIESES, AJUSTANDO CONDUTAS}

$\mathrm{Na}$ antropologia, uma das mais importantes discussões acerca do comportamento econômico e a natureza humana remete aos trabalhos de Karl Polanyi (I 944, I 977). O autor não apenas critica o modelo de Homo oeconomicus, como também propõe uma reflexão sobre a primazia da ideia de racionalidade na atual sociedade.

A partir de trabalhos etnográficos voltados para a compreensão das sociedades primitivas (Malinowski, I984; Mauss, 2003; Firth, I929; Thurnwald, 20I8), Polanyi reprova as interpretações de teóricos econômicos neoclássicos sobre a organização econômica das sociedades. Em seu argumento, defende que as sociedades pré-capitalistas não deveriam ser entendidas como uma forma rudimentar da sociedade de mercado, assim como os comportamentos baseados na maximização dos ganhos e nos cálculos de custo/ 
oportunidade não deveriam ser concebidos como inatos ao humano. ${ }^{12}$ Como lembra o autor, as motivações econômicas poderiam ser orientadas por elementos como religião, obrigações para com a comunidade, poder político, ideais de honra, padrões estéticos etc. - motivos bastante distintos da procura por ganhos individuais descritos como lucro. Polanyi (I977: 5) denomina, assim, "falácia economicista" essa insistência em igualar a economia humana a sua forma mercado (isto é, aquela marcada pelos mecanismos competitivos de oferta-demanda-preço); tal equivalência era entendida como um erro, uma vez que a economia humana seria muito mais rica.

As críticas de Polanyi (I977) se dão em direção à arbitrariedade dessas definições tomadas como universais. Apesar de reconhecer o triunfo do racionalismo econômico, considerava um mito reduzir toda a vida humana a indivíduos que se comportam dentro da lógica de mercado.

Vale, entretanto, ressaltar que essas posições de Polanyi são distintas do modo como a economia comportamental vem construindo as críticas ao Homo oeconomicus. Verifica-se que esta última preserva a ideia de racionalidade econômica. Apesar das frequentes críticas ao conceito de agente econômico racional, egoísta e maximizador de seus interesses, esse modelo não é descartado, mas humanizado.

Como bem descreve Bergeron et al. (2018), mesmo que pesquisadores critiquem o conceito de Homo oeconomicus como uma abstração teórica afas tada da realidade e admitam que as pessoas são seres humanos falhos (que se confundem, agem de forma intuitiva e cometem erros sistemáticos), a abordagem empiricamente verificável da economia comportamental não se impõe necessariamente como uma ruptura com o modelo neoclássico, uma vez que o comportamento racional é mantido como quadro de referência. Nessa perspectiva, a racionalidade humana seria naturalmente falha, cabendo à economia comportamental corrigir ou explorar esses defeitos para fazer as ações individuais convergirem a um modelo ideal, bastante próximo da racionalidade do Homo oeconomicus.

Logo, a economia comportamental pode ser entendida como complementar às teorias econômicas neoclássicas, oferecendo subsídios capazes de aprimorá-las. Como propõe Richard Thaler (20I6), o conjunto de dados com que trabalham os economistas poderia ser enriquecido a partir de experimentos de laboratórios, dos dados colhidos em campo ou das informações obtidas a partir de imagens cerebrais. Seguindo o raciocínio do autor, a economia comportamental não deveria ser interpretada como uma "revolução", mas como o começo do desenvolvimento de teorias que levem em conta agentes humanos, pessoas reais.

A perspectiva de Thaler reforça a argumentação de Callon (I998: 22) de que o Homo oeconomicus, de fato, existe. Tal existência, no entanto, não deve ser interpretada como parte da natureza humana, mas como o resul- 
tado de processos de configuração, em que investimentos de toda sorte são feitos a fim de conformar e equipar os agentes, de forma que eles possam se aproximar da racionalidade econômica desejada. Nesse sentido, é valido reforçar que são justamente as práticas, os saberes e as ferramentas desenvolvidos para modelar esse Homo oeconomicus que continuam chamando a atenção e tornando-se objeto de reflexão dos pesquisadores da antropologia da economia.

Ao analisar o desenvolvimento da Enef, ficou nítido que somadas às iniciativas educativas ganham espaço as experiências que utilizam nudges. Frente às controvérsias sobre os reais resultados das inciativas pedagógicas voltadas para a educação financeira, as ciências comportamentais apresentam novas modalidades de intervenção para governar as condutas das pessoas. Nessa trajetória, eventos como a Conferência descrita neste texto funcionam como uma espécie de incubadora - um espaço para divulgação e legitimação das novas abordagens metodológicas - com a função de aproximar a academia e gestores de políticas públicas, sugerindo e estimulando os especialistas que ultrapassem as iniciativas pedagógicas. Se cada vez mais o cérebro passa a responder por fatores anteriormente atribuídos ao indivíduo, ao ambiente e à sociedade (Ortega, 2009; Rose \& Abi-Rached, 2013), vale estar atento para acompanhar os tipos de intervenções que surgirão a partir dele.

Recebido em 29/05/2020 | Revisto em 08/I 2/2020 | Aprovado em 07/0I/202 I

Viviane Fernandes é doutora em antropologia social pelo Programa de Pós-Graduação em Antropologia Social do Museu Nacional (UFRJ) e atualmente realiza estágio pós-doutoral na mesma instituição. É pesquisadora do Núcleo de Pesquisas em Cultura e Economia (NuCEC). 


\section{NOTAS}

I Em 20I7, após aprovação da fusão entre BM\&FBovespa e Cetip, a nova companhia de infraestrutura de mercado financeiro passa a ser conhecida como B3 (Brasil, Bolsa, Balcão).

2 Para conhecer mais sobre o trabalho de formação de investidores, ver Leite $(2016,2017)$.

3 Lançada em dezembro de 20Io, pelo decreto n. 7.397 do governo federal, a Enef é uma política de Estado que tem como objetivo promover a educação financeira e previdenciária no país.

4 A pesquisa de Mauss e Hubert (2003) no intuito de desenvolver uma teoria geral da magia servia-me de inspiração metodológica nesses primeiros passos do estudo. Ainda buscando compreender o que eram e como se desenvolviam as iniciativas de educação financeira, comecei a catalogar suas definições e a reconhecer seus elementos (atores, práticas e representações). Meu objetivo era consolidar uma série de informações a fim de ter uma imagem mais completa de como vinha sendo desenvolvida a política em suas muitas frentes. Como eram pouquíssimos os estudos sobre educação financeira no país, busquei organizar as diferentes informações que coletava (a partir de artigos, matérias de jornais, projetos de lei, eventos, entrevistas, planos de implementação de política pública) seguindo o mesmo formato de organização dos dados utilizados pelos autores - (a) históricos e fontes; (b) definição, (c) elementos e (d) análise e explicação.

5 Natasha Schüll e Caitlin Zaloom (20I I) exploram o recente campo da neuroeconomia. As autoras apresentam o crescimento das discussões e controvérsias em torno das atividades cerebrais e a possibilidade de intervenção governamental nos problemas de escolha humana. Já a dissertação de Guilherme Giufrida (20I5) também se dedica a compreender os avanços dos "estudos da mente", apresentando o desenvolvimento da psicologia econômica, da neuroeconomia e do neuromarketing no Brasil.

6 Banco Central do Brasil (BCB), Comissão de Valores Mobiliários (CVM), Superintendência Nacional de Pre- 
vidência Complementar (Previc) e Superintendência de Seguros Privados (Susep) são os quatro reguladores do Sistema Financeiro Nacional que estão à frente da elaboração e condução da Enef.

7 Para crianças e adolescentes, a implementação da Enef foi estimulada por intermédio das escolas. O material diádico é composto por um conjunto de livros produzido especificamente para cada ano do ensino fundamental e médio.

8 A conferência ocorre anualmente no segundo semestre do ano. Em 2020, foi realizada sua oitava edição. O histórico da programação e das apresentações realizadas pode ser encontrado no site <http://www.iecbrazil. com.br/>.

9 Os trabalhos de Brien Moeran, sobre feiras de livros, e Lise Skov, sobre feiras de negócios de moda, também chamam a atenção para o fato de esses eventos funcionarem como "pontos nodais" (Moeran, 20Io), sendo capazes de reunir e colocar em relação atores que estão geograficamente dispersos (Skov, 2006).

Io Apesar de se concentrar em um universo bastante distinto, John Comerford (I999: 46) interpreta as reuniões como elemento importante na produção de um espaço de sociabilidade e de consolidação de redes de relações. Essa interpretação é bastante produtiva para examinar os eventos/conferências de educação financeira. Apoiada na perspectiva do autor, compreendo que, para além de uma dimensão prática - relativa à disseminação de conteúdo -, esses eventos também são importantes para construir um universo social no qual são reunidos agentes, compartilhadas teorias, apresentadas ferramentas, legitimadas determinadas práticas e, consequentemente, consolidada a própria política de educação financeira.

I I Para uma discussão mais detalhada sobre a relevância do networking em eventos, ver o trabalho de Monise Picanço (20I9), que ao abordar as feiras de negócios discute a importância da circulação dos participantes na construção de relacionamentos.

I2 No capítulo The descente of economic man, Gareth Dale (2010) apresenta as críticas de Polanyi à economia clássica e apresenta os conceitos de economia substantivista e formalista desenvolvidos pelo autor. 


\section{REFERÊNCIAS BIBLIOGRÁFICAS}

Bergeron, Henri et al. (2018). Le biais comportementaliste. Paris: Press de Science Po.

Brasil. (20Io). Estratégia nacional de educação financeira: plano diretor. Brasília: Conef.

Callon, Michael. (I998). The law of markets. Oxford: Blackwell.

Comerford, John. (1999). Fazendo a luta: sociabilidade, falas e rituais na construção de organizações camponesas. Rio de Janeiro: Relume Dumará/Núcleo de Antropologia da Política.

Dale, Gareth. (2010). Karl Polanyi: The limits of the market. Cambridge: Polity Press.

Fernandes, Daniel; Lynch, John \& Netemeyer, Richard. (20I4). Financial literacy, financial education, and downstream. Financial Behaviors. Management Science, 6o/8.

Fernandes, Viviane. (20I9). Cuidando da saúde financeira: uma etnografia sobre endividamento. Tese de Doutorado. PPGAS/ Universidade Federal do Rio de Janeiro.

Firth, Raymond. (1929). Primitive economics of the New Zealand Maori. London: George Routledge.

Friedman, Milton. (1957). Theory of the consumption function. New Jersey: Princeton University Press.

Giufrida, Guilherme. (20I5). Exatas e humanas: uma antropologia dos estudos da mente e da economia. Dissertação de Mestrado. PPGAS/Universidade Federal do Rio de Janeiro.

Jevons, William. (I875). Money and the mechanism of exchange. New York: D. Appleton and Co.

Kahneman, Daniel. (20I I). Thinking, fast and slow. New York: Farrar, Straus and Giroux.

Kaiser, Tim \& Menkhoff, Lukas. (2016). Does financial education impact financial behavior, and if so, when? Discussion Paper, I562.

Leite, Elaine Silveira. (2017). A ressignificação da figura do especulador-investidor e as práticas de educação financeira. Civitas - Revista de Ciências Sociais, I7/I.

Leite, Elaine Silveira. (2016). Reconversão de habitus: o advento do ideário de investimento no Brasil. São Carlos, 20I I. Tese de Doutorado. PPGS/Universidade Federal de São Carlos. 
Lusardi, Annamaria. (2003). Saving and the effectiveness of financial education. Pension Research Council WP2003-I4. Disponível em: $\langle$ https://ssrn.com/abstract $=47602>$. Aces so em 20 abr. 2019.

Malinowski, Bronislaw. (I984) [1922]. Argonautas do Pacífico Ocidental. São Paulo: Abril.

Mauss, Marcel. (2003) [1924]. Ensaio sobre o dom. In: Mauss, Marcel. Sociologia e antropologia. São Paulo: Cosac \& Naify. Mauss, Marcel \& Hubert, Henri. (2003). Esboço de uma teoria geral da magia. In: Mauss, Marcel. Sociologia e antropologia. São Paulo: Cosac \& Naify.

Miller, Margaret et al. (20I5). Can you help someone become financially capable? A meta-analysis of the literature. World Bank Research Observer, 30/2, p. 220-246.

Moeran, Brian. (2010). The book fair as a tournament of values. The Journal of the Royal Anthropological Institute, I6/I.

Ortega, Francisco. (2009). Neurociências, neurocultura e autoajuda cerebral. Interface - Comunicação, Saúde, Educação, I3/3I, p. 247-260.

Picanço, Monise. (20I9). Caleidoscópio da valoração. A HSM Expomanagement e o processo de constituição de seus produtos. Tese de Doutorado. PPGS/Universidade de São Paulo.

Polanyi, Karl. (1977). The livelihood of man. New York: Academic Press.

Polanyi, Karl. (I944). The great transformation. New York/ Toronto: Farrar \& Rinehart.

Rose, Nikolas \& Abi-Rached, Joelle. (2013). Neuro: the new brain sciences and the management of the mind. Princeton: Princeton University Press.

Schüll, Natasha \& Zaloom, Caitlin. (20II). The shortsighted brain: neuroeconomics and the governance of choice in time. Social Studies of Science, $4 \mathrm{I} / 4$.

Simon, Herbert. (I982). Models of bounded rationality. Cambridge: MIT Press.

Simon, Herbert. (I955). A behavioral model of rational choice. The Quarterly Journal of Economics, 69/I.

Skov, Lise. (2006). The role of trade fairs in the global fashion business. Current Sociology, 54/5. 
Soares, Fabrício. (2017). Os debates sobre a educação financeira em um contexto de financeirização da vida doméstica, desigualdade e exclusão financeira. Tese de Doutorado. PPGCS/Pontifícia Universidade Católica do Rio de Janeiro. Sunstein, Robert. (2014). Nudging: a very short guide. Journal of Consumer Policy, 37, p. 583-588.

Thaler, Richard. (20I6). Behavioral economics: past, present and future. American Economic Review, 106/7.

Thaler, Richard. (I999). Mental accounting matters. Journal of Behavioral Decision Making, I2.

Thaler, Richard. (1980). Toward a positive theory of consumer choice. Journal of Economics Behavior and Organization, 39 .

Thaler, Richard \& Shefrin, Hersh. (I98I). An economic theory of self-control. Journal of Political Economics, 89/2.

Thaler, Richard \& Sustein, Cass. (2008). Nudge: improving decisions about health, wealth, and happiness. New Haven: Yale University Press.

Thurnwald, Richard. (2018) [1932]. Economics in primitive communities. London: Routledge.

Tversky, Amos \& Kahneman, Daniel. (I979). Prospect theory: an analysis of decision under risk. Econometrica, $47 / 2$.

Tversky, Amos \& Kahneman, Daniel. (1974). Judgment under uncertainty: heuristics and biases. Science, I85/4I57. 


\section{DA EDUCAÇÃO AO EMPURRÃO: A PARTICIPAÇÃO DAS CIÊNCIAS COMPORTAMENTAIS EM PROGRAMAS DE EDUCAÇÃO FINANCEIRA \\ Resumo}

No formato de registro de pesquisa o artigo explora em que contexto as ciências comportamentais ganham espaço dentro das iniciativas de educação financeira no país. A partir de uma abordagem etnográfica, examino a importância da Conferência de Educação Financeira e Comportamento do Investidor como ferramenta para reunir agentes dispersos e conferir legitimidade e congruência a um conjunto de teorias que - na intersecção da psicologia e economia - discutem os mecanismos da tomada de decisões econômicas.

FROM EDUCATION TO NUDGE: BEHAVIORAL SCIENCES IN FINANCIAL EDUCATION PROGRAMS

Abstract

This paper explores in which context the behavioral sciences become relevant to financial education initiatives in the country. From an ethnographic approach, I examine the importance of the Financial Education and Investor Behavior Conference as a tool to bring together dispersed agents and confer legitimacy and congruence to a set of theories that - at the intersection of psychology and economics - discuss the mechanisms of deci-

\section{Palavras-chave}

Educação financeira;

ciências comportamentais;

homo oeconomicus;

política pública.

Keywords

Financial education;

behavioral sciences; homo oeconomicus; public policy. 